\title{
Respuesta geomórfica de los ríos Tulín y Turrubaritos a la deformación asociada la falla Angostura, antearco central de Costa Rica
}

\author{
Geomorphic response of the rivers Tulin and Turrubaritos to \\ the deformation associated with the angostura fault, a Central \\ Forearc in Costa Rica
}

\author{
Hernán Porras-Espinoza ${ }^{1}$ \\ Universidad Nacional, Costa Rica \\ Carolina Fallas-Salazar ${ }^{2}$ \\ Kevin Stanlyn Sarmiento ${ }^{3}$ \\ Mario Arroyo-Solorzano ${ }^{4}$ \\ Universidad de Costa Rica, Costa Rica
}

\section{Resumen}

La falla Angostura ubicada en el antearco central externo de Costa Rica, es una falla normal que presenta un sistema de relevo mediante una rampa. Esta falla implantada dentro del basamento asociado a la Formación Tulin estuvo activa durante el Mioceno Inferior. Generando así, una serie de depocentros dentro de los cuales se habrían depositado los sedimentos de la Formación Punta Carballo. Los índices geomórficos, así como el análisis del patrón de drenaje y de los paleocanales,

1 Observatorio Vulcanológico y Sismológico de Costa Rica, Universidad Nacional, Costa Rica. Correo electrónico: porrashernan@gmail.com

2 Escuela Centroamericana de Geología, Universidad de Costa Rica. Correo electrónico: caro.fallas.s@gmail.com

3 Escuela Centroamericana de Geología, Universidad de Costa Rica. Correo electrónico: sstanlyn.1@gmail.com

4 Escuela Centroamericana de Geología, Universidad de Costa Rica. Correo electrónico: marroso1210@gmail.com 
Hernán Porras-Espinoza, Carolina Fallas-Salazar, Kevin Stanlyn Sarmiento, Mario Arroyo-Solorzano. Geomorphic response of the rivers Tulin and Turrubaritos to the deformation associated with the angostura fault, a Central Forearc in Costa Rica

sugieren que esta falla ha contribuido activamente en el modelado del paisaje, sin embargo, no presenta actividad al menos desde el Mioceno Medio al presente.

Palabras clave: Tectónica, geomorfología, índices geomórficos, antearco central de Costa Rica.

\begin{abstract}
The Angostura fault, located in the central outer forearc of Costa Rica, is a normal fault that presents a relay system using a ramp. This fault, implanted within the basement associated with the Tulin Formation, was active during the Early Miocene, thus generating a series of depocenters within which sediments of the Formación Punta Carballo would have been deposited. The geomorphic indices, as well as the analysis of the pattern of drainage and the paleochannels, suggest that this fault has actively contributed in the modeling of the landscape; however, it does not present activity since, at least, the Middle Miocene to the present.
\end{abstract}

Keywords: Tectonics, geomorphology, geomorphic indices, central forearc of Costa Rica

\title{
Introducción
}

Costa Rica se encuentra en el suroeste de la Placa Caribe y el noreste de la trinchera centroamericana. La costa del Pacífico de Costa Rica se deforma por la subducción de la Placa Coco bajo la Placa del Caribe (Figura 1) (Protti, 1995; DeMets et al., 2001), desarrollando una deformación significativa en el antearco a través del tiempo (Fisher et al., 2004; Sak et al., 2009; Morell et al., 2012; Mescua et al., 2017). 
Hernán Porras-Espinoza, Carolina Fallas-Salazar, Kevin Stanlyn Sarmiento, Mario Arroyo-Solorzano. Respuesta geomórfica de los ríos Tulín y Turrubaritos a la deformación asociada

la falla Angostura, antearco central de Costa Rica

Figura 1: Mapa de ubicación con las principales características morfotectónicas, recuadro blanco indica la zona de estudio ubicada en el antearco central.

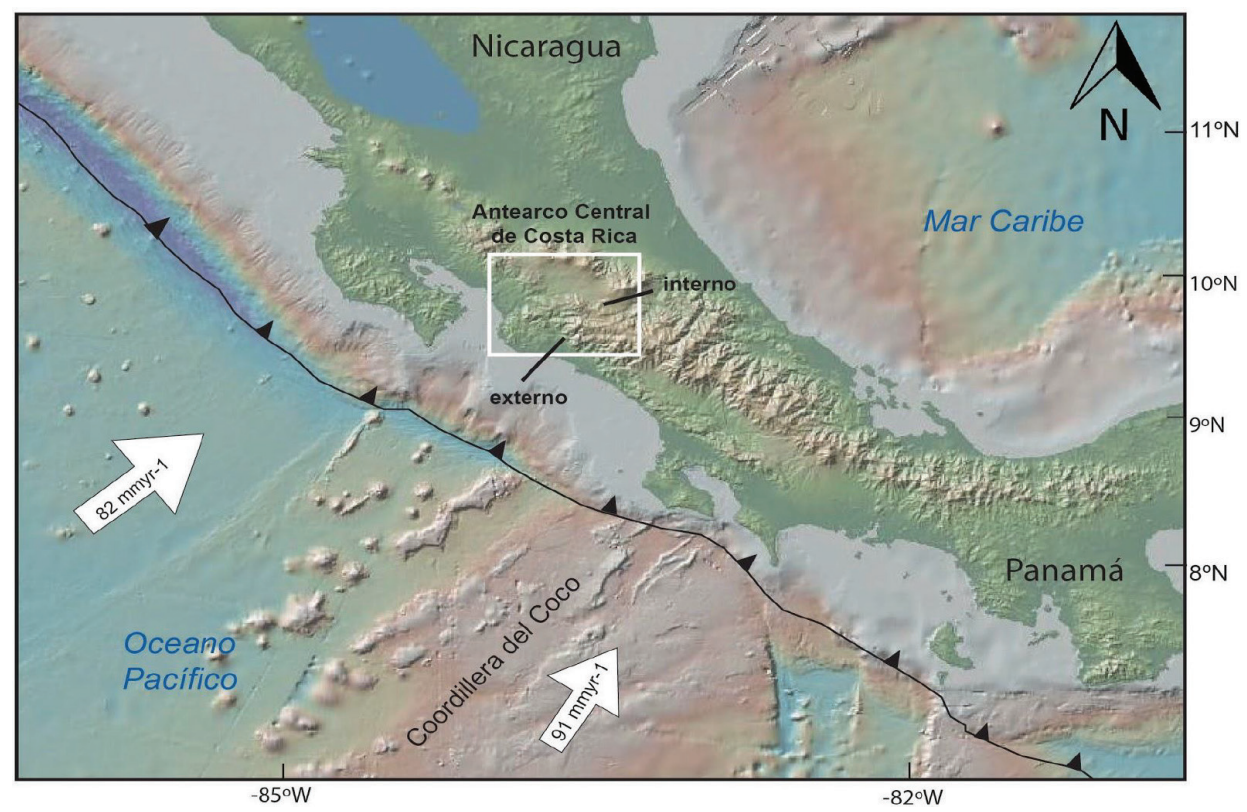

Fuente: Imagen tomada de Geomaapp ${ }^{\circ}$.

El antearco central de Costa Rica es un laboratorio natural para el estudio de la morfología de los cauces y su relación con la tectónica. Esta zona de antearco se subdivide en: antearco interno (Figura 1), con predominancia de rocas sedimentarias y volcanoclásticas del Neógeno (Figura 2) (Denyer y Arias, 1991); antearco externo, caracterizado por la presencia de rocas volcánicas y sedimentarias del Paleógeno y Neógeno (Arias, 2000).

En el antearco externo se han reportado una serie de fallas activas (Denyer y Alvarado, 2007) entre las que se encuentra una sin nombre que se ha denominado falla Angostura (Figura 2). El trazo de esta tiene una longitud mínima de aproximadamente $15 \mathrm{~km}$ y un desplazamiento dextral, según Denyer y Alvarado (2007). Se considera probable que esta falla sea una fuente sismogénica (Denyer et al., 2003) y represente un riesgo sísmico considerable para la región del Pacífico central. Sin embargo, debido a la falta de estudios que involucren mediciones cuantitativas y análisis de 
Hernán Porras-Espinoza, Carolina Fallas-Salazar, Kevin Stanlyn Sarmiento, Mario Arroyo-Solorzano. Geomorphic response of the rivers Tulin and Turrubaritos to the deformation associated with the angostura fault, a Central Forearc in Costa Rica

índices geomórficos, la actividad tectónica de la falla Angostura no se ha definido previamente.

Figura 2: Mapa geológico del antearco central. El área del mapa corresponde al recuadro blanco de la figura 1. Modificado de Denyer y Alvarado (2007). FJ, falla Jaris. FC, falla Candelaria. FA, falla Angostura. Fallas no comprobadas en línea roja.

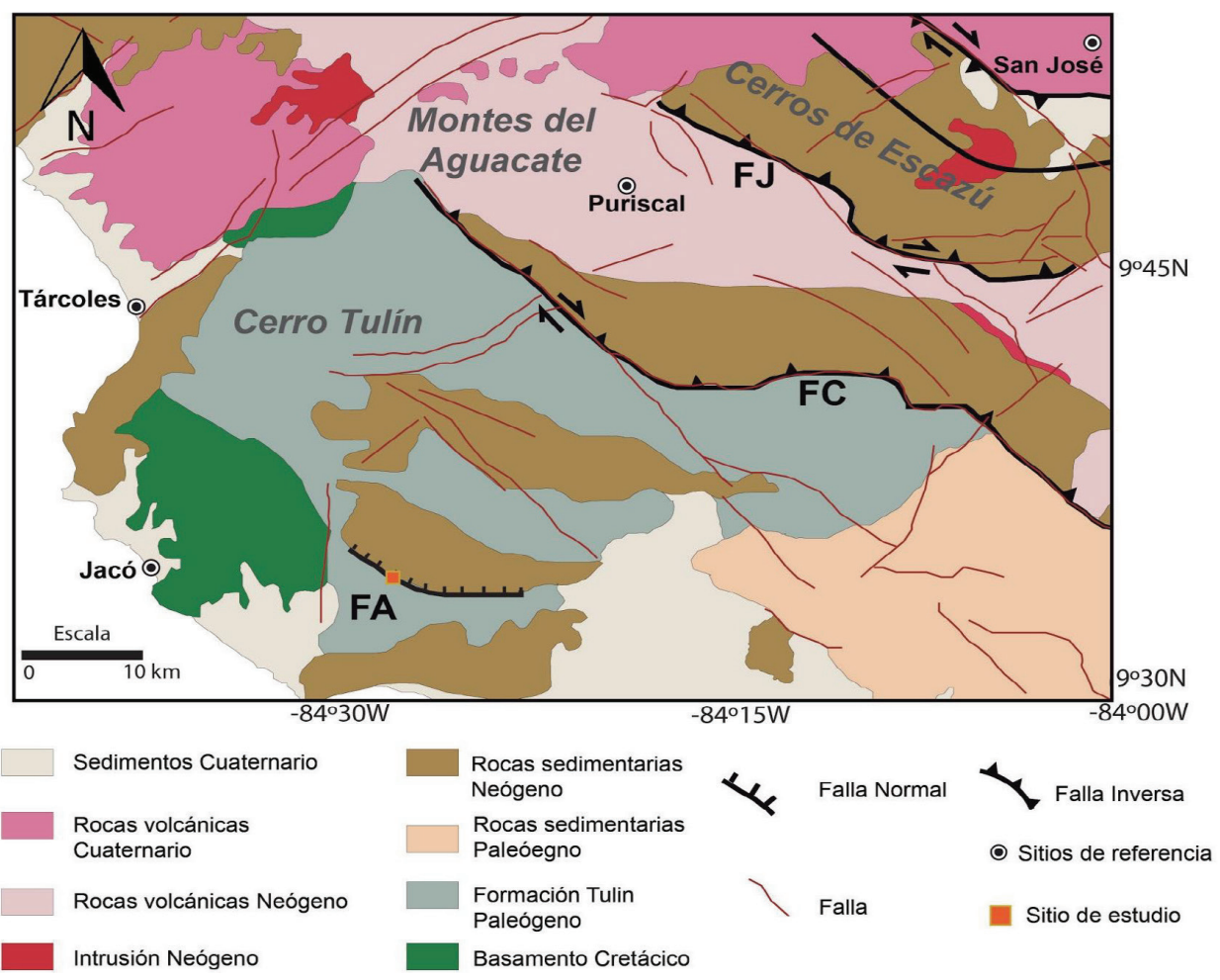

Fuente: elaborada por los autores.

Por otra parte, la interacción entre tectónica activa y procesos superficiales origina la formación de marcadores geomórficos mensurables a lo largo de fallas. Es así, como la geomorfología tectónica se ha convertido en una de las principales herramientas utilizadas en el estudio de la tectónica activa y en la evolución del paisaje (Bull, 2007; Burbank y Anderson, 2001; Keller y Pinter, 2002). 
Hernán Porras-Espinoza, Carolina Fallas-Salazar, Kevin Stanlyn Sarmiento, Mario Arroyo-Solorzano. Respuesta geomórfica de los ríos Tulín y Turrubaritos a la deformación asociada

la falla Angostura, antearco central de Costa Rica

El alzamiento y subsidencia de bloques producido por fallas en los valles, exhibe marcadores geomorfológicos, y el uso de mediciones cuantitativas de las características, tales como sistemas de drenaje, sinuosidades del frente de montaña, entre otros, juegan un papel destacado en la revelación de la actividad tectónica (Keller, 1986; Keller y Pinter, 2002; entre otros).

Este estudio presenta, por lo tanto, un análisis cinemático y geomórfico de la región de antearco externo, precisamente por donde se sugiere la traza de la falla Angostura, con el fin de evaluar su actividad tectónica. Se ha examinado la dinámica entre el frente montañoso y las características morfológicas de los ríos más importantes de esta zona, el río Tulin y el río Turrubaritos. El análisis realizado se basa en el trabajo de campo y en la interpretación de la morfología del cauce de los principales ríos que atraviesan la zona. De esta manera, se pretende demostrar la presencia de movimientos activos o no a lo largo de la falla Angostura.

\section{Geología regional y tectónica}

Durante el Cretácico Superior y el Cenozoico, el régimen de subducción a lo largo del margen occidental de la Placa del Caribe proporcionó los controles de primer orden sobre la subsidencia y elevación en el área, definiendo así su evolución tectónica (Mann, 2007; Mescua et al., 2017). El antearco central de Costa Rica tiene un marco estructural complejo. Durante el Cenozoico temprano, el margen suroeste de la Placa del Caribe estaba marcado por una trinchera activa, dos arcos magmáticos relativamente angostos (Bundschuh y Alvarado, 2007; Saginor et al., 2013) y una serie de cuencas sedimentarias extensionales (Astorga et al., 1991; Barboza et al., 1995).

En la zona de estudio, las cuencas de Candelaria y Térraba se desarrollaron durante el Cenozoico temprano en el margen del Pacífico Central de Costa Rica, caracterizadas por depocentros con tendencia noroeste-sureste, implantados en la corteza mesozoica (Astorga et al., 1991). Durante el Mioceno medio, la dirección de subducción es esencialmente ortogonal a la trinchera, iniciando así un periodo contraccional en la Placa superior (Mescua et al., 2017). Esta característica ha causado una serie de rasgos geológicos en Costa Rica, incluyendo la falta de volcanismo de arco activo, elevación de la Cordillera Talamanca y el acortamiento del arco hacia 
Hernán Porras-Espinoza, Carolina Fallas-Salazar, Kevin Stanlyn Sarmiento, Mario Arroyo-Solorzano. Geomorphic response of the rivers Tulin and Turrubaritos to the deformation associated with the angostura fault, a Central Forearc in Costa Rica

atrás a lo largo del cinturón deformado del norte de Panamá (Norabuena et al., 2004; Mescua et al., 2017).

En el presente, la Placa Coco se subduce bajo la Placa del Caribe a una tasa de convergencia de $91 \mathrm{mma}^{-1}$ (Bird, 2003; DeMets, 2001) a 82 $\mathrm{mma}^{-1}$ (DeMets, 2010). Estudios de velocidad geodésica (LaFemina et al., 2009), terrazas marinas (Gardner et al., 2001; Sak et al., 2004, 2009), mapeo geológico y geomorfológico (Fisher et al., 2004; Sitchler et al., 2007), han documentado el acortamiento y la elevación en el antearco de Costa Rica.

\section{Metodología}

En la geomorfología tectónica se trata de señalar características o superficies geomórficas identificables que proporcionen una referencia contra la cual medir la deformación diferencial o absoluta, estas características son llamadas indicadores o marcadores geomórficos.

Los datos de los índices geomórficos para los análisis del patrón de drenaje se obtuvieron de factores como la topografía y modelos de elevación digital (DEM, por sus siglas en inglés) en la zona, análisis de fotos e imágenes orbitales y del trabajo de comprobación de campo. Además de estos aspectos, meramente geomorfológicos, se tomaron datos estructurales, litológicos y sísmicos que permiten complementar el análisis con un componente de análisis cinemático importante.

Los índices geomorfológicos incluyen patrones axiales de ríos, índices de frente de montaña y facetas triangulares. Las mediciones cuantitativas y el análisis de dichos índices se utilizaron para evaluar la actividad tectónica de la falla Angostura. Según Burbank y Anderson (2001) un frente de montaña con tectónica activa suele caracterizarse por una serie de rasgos morfotectónicos, tales como; frente de montaña lineal, grandes facetas y ríos axiales proximales, que son típicamente creados por el hundimiento rápido de la pared colgante (hanging wall) y el levantamiento relativo del bloque de piso (foot wall). El análisis morfométrico del frente de montaña se hizo mediante el uso de índices geomorfológicos de sinuosidad del frente de montaña $\left(\mathrm{S}_{\mathrm{fm}}\right)$ y facetas triangulares, con el objetivo de evaluar la interacción entre las fuerzas de erosión y las fuerzas tectónicas en esta área.

El valor del $\mathrm{S}_{\mathrm{fm}}$, se obtiene por medio de la relación entre la longitud del frente de montaña $\mathrm{L}_{\mathrm{fm}}$ y la longitud de la línea recta que une los dos extremos del frente montañoso $\mathrm{L}_{\mathrm{s}}$, por lo tanto: $\mathrm{S}_{\mathrm{fm}}=\mathrm{L}_{\mathrm{fm}} / \mathrm{L}_{\mathrm{s}}($ Bull, 2007). 
Hernán Porras-Espinoza, Carolina Fallas-Salazar, Kevin Stanlyn Sarmiento, Mario Arroyo-Solorzano.

Respuesta geomórfica de los ríos Tulín y Turrubaritos a la deformación asociada

la falla Angostura, antearco central de Costa Rica

En el análisis de las facetas triangulares, este se determina de acuerdo con la proporción de facetas a lo largo del frente de montaña (Lf / Ls, donde Lf es la topografía de las facetas y Ls la longitud lineal de las facetas).

También se trabajó sobre aspectos como el sistema y patrón de drenaje de los ríos, así como con el análisis respectivo de los perfiles longitudinales de los mismos y de las terrazas. Para el análisis del patrón de drenaje que incluye el río axial del sistema de drenaje, Holbrook y Schumm (1999), sugieren que los cambios en las variables hidráulicas del comportamiento de los ríos ocurren a medida que las corrientes cruzan zonas de elevación o subsidencia que impactan en la forma transversal de las mismas.

Los valores se determinaron usando mapas topográficos en una escala de 1: 50,000 y un DEM basado en estos mapas. Además, se utilizaron imágenes de satélite archivadas por Google Earth ${ }^{\mathrm{TM}}$, y un mapa topográfico, con el fin de determinar la posición relativa de los paleocanales y los arcos (formados por el límite del meandro) de la corriente del rio Tulin y Turrubaritos.

\section{Análisis Cinemático de los datos de deslizamiento de la falla}

En el sector suroeste de la falla Angostura se ubica una zona de daño donde es posible obtener indicadores cinemáticos asociados a su movimiento. El análisis de estos datos muestra que se trata de una falla normal con un deslizamiento casi puro, con un plano de inclinación medio de aproximadamente $45^{\circ}$ hacia el Noreste (Figura 3). 
Hernán Porras-Espinoza, Carolina Fallas-Salazar, Kevin Stanlyn Sarmiento, Mario Arroyo-Solorzano. Geomorphic response of the rivers Tulin and Turrubaritos to the deformation associated with the angostura fault, a Central Forearc in Costa Rica

Figura 3: Cinemática de la Falla Angostura, tomados a partir de fracturas con intercrecimientos de fibras minerales.

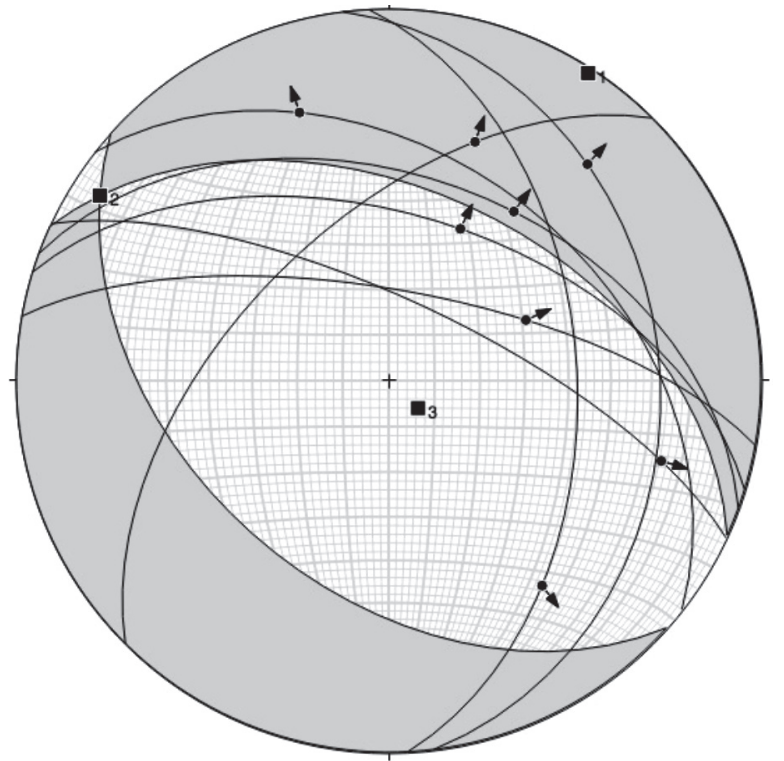

Fuente: Figura elaborada por los autores utilizando el software faultkin de Allmendinger y Cardoso.

Para obtener una mejor interpretación de la falla Angostura, se aproximó con base en los datos de indicadores cinemáticos recolectados en el campo, diagramas de rosas para la aproximación geométrica de la falla. Se introdujeron las mediciones estructurales al programa Wintensor, teniendo así una mejor base estadística y mayor criterio para aproximar aspectos como el Rumbo, dirección y ángulo de inclinación, el pitch o rake y el esfuerzo máximo horizontal y relacionar esto con el contexto tectónico de la zona (Figura 4). 
Hernán Porras-Espinoza, Carolina Fallas-Salazar, Kevin Stanlyn Sarmiento, Mario Arroyo-Solorzano. Respuesta geomórfica de los ríos Tulín y Turrubaritos a la deformación asociada

la falla Angostura, antearco central de Costa Rica

Figura 4: Resultados de análisis estadístico de los indicadores cinemáticos de la Falla Angostura
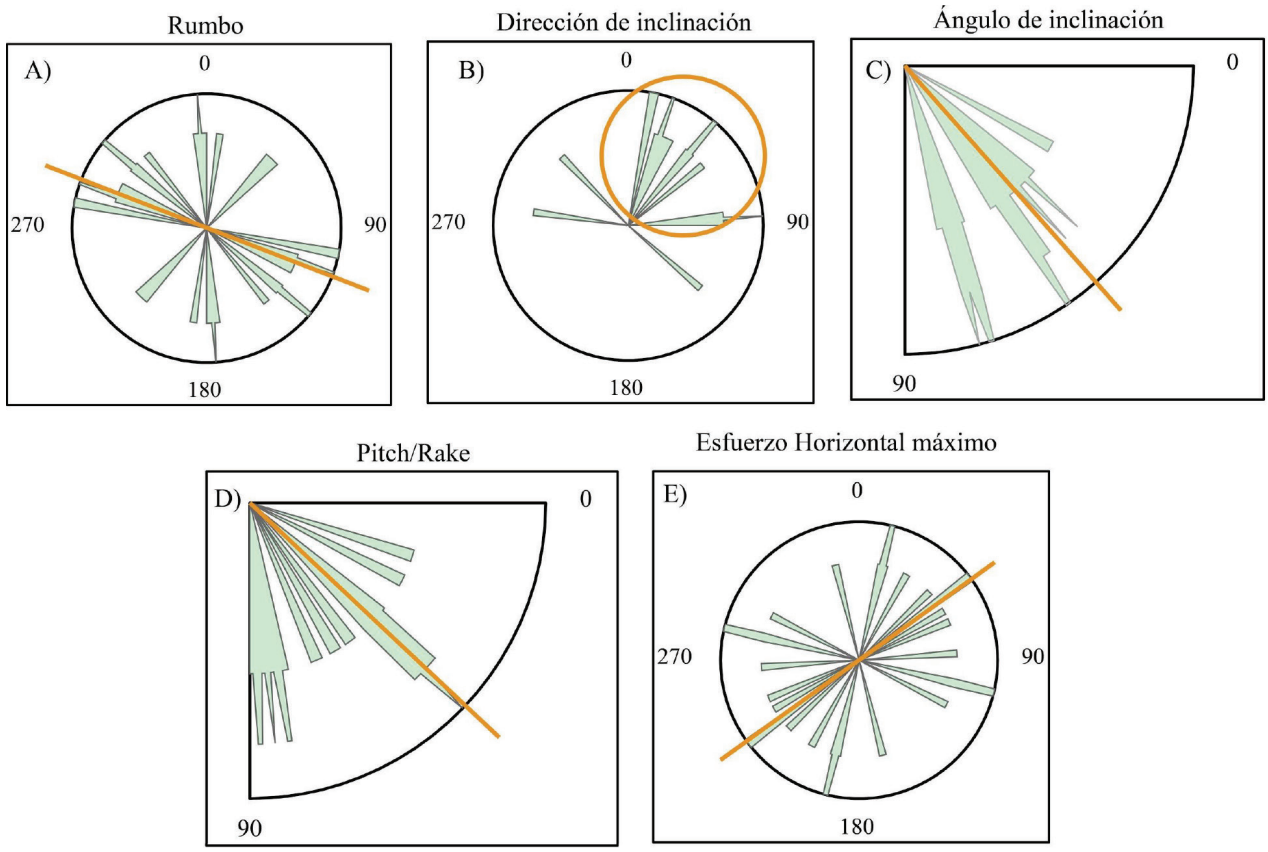

Esfuerzo Horizontal máximo

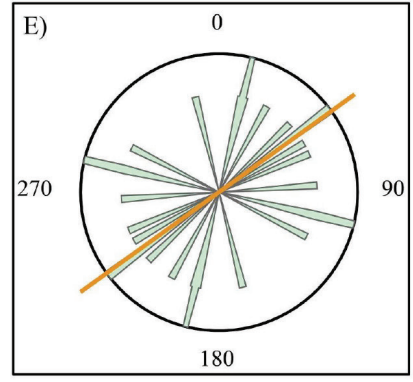

Fuente: Figura elaborada por los autores utilizando el software Win-Tensor de Delvaux.

Este análisis evidencia que el rumbo más probable para la falla sigue predominantemente una dirección noroeste-sureste (Figura 4.A), lo cual coincide con el trazo propuesto y la dirección de los escarpes en el mapa del área de estudio. La dirección de inclinación de las fallas, apunta a tener sentido Noreste (Figura 4.B) y el ángulo de inclinación predominantemente se muestra entre $20^{\circ}$ a $60^{\circ}$, lo que apoya un sentido normal de las estructuras, por la tendencia hacia un alto ángulo (Figura 4.C). El ángulo de rake o pitch, si bien presenta múltiples posibilidades, tiene una predominancia de $45^{\circ}$, los altos ángulos demuestra la presencia de un componente vertical, el cual relacionamos con fallamiento normal (Figura 4.D). Por último, la dirección del esfuerzo máximo horizontal es mayoritariamente en un sentido suroeste-noreste, lo cual concuerda totalmente con lo esperado, según el régimen tectónico de Costa Rica, de acuerdo con el sentido de la subducción y la ubicación de la zona relativamente cercana a la trinchera (Figura 4.E). 
Hernán Porras-Espinoza, Carolina Fallas-Salazar, Kevin Stanlyn Sarmiento, Mario Arroyo-Solorzano. Geomorphic response of the rivers Tulin and Turrubaritos to the deformation associated with the angostura fault, a Central Forearc in Costa Rica

Los resultados calculados definen una aproximación vertical del sigma 1 con una inclinación hacia el sureste de alrededor de $80^{\circ}$. El sigma 2 es sub-horizontal a $10^{\circ}$ de inclinación al noreste. El sigma 3 es casi en la horizontal en la dirección noreste (Figura 3).

Con respecto a la sismicidad instrumental registrada en Costa Rica, la zona de estudio se ubica muy próxima a la trinchera y en el sector del Pacífico costarricense (el más activo sísmicamente). Rodeada de zonas con sismicidad muy importante, como Parrita y Quepos, que se ubican entre los cinco cantones más sísmicos de Costa Rica y entre los cúmulos sísmicos asociados a la subducción de los montes submarinos como el Plateau de Quepos y el Fisher seamount (Godínez et al., 2018 “in press"). Sin embargo, la zona en específico, se muestra como un gap o vacío sísmico en cuanto a sismicidad importante (mayor a $5 \mathrm{Mw}$ ), lo cual se evidencia en Arroyo et al., 2017, donde al estudiar la sismicidad mayor a 5Mw por décadas, se aprecia la no actividad sísmica de dicha índole, aun estando rodeado de grandes sismos como en la década de los 90, con los enjambres de Puriscal, el sismo de Cóbano en 1990 y Quepos 1999.

\section{Índices Geomórficos}

\section{Frente de montaña, sinuosidad frontal de la montaña (Sfm) y facetas triangulares}

Durante los estudios de campo, se observan dos prominentes escarpes y una zona de relevo asociados al movimiento de la Falla Angostura (Figura 5). Estas morfoestructuras marcan el límite entre los basaltos asociados al basamento Cretácico y la cobertura sedimentaria del Mioceno Inferior-Medio. El relleno de la cuenca estructural actual subyacente consiste principalmente de aluviones depositados por los ríos Tulin y Turrubaritos y coluvios derivados de los escarpes de falla.

El índice de $\mathrm{S}_{\mathrm{fm}}$ se calcula para el frente de la montaña, entre los ríos Tulin y Turrubaritos a lo largo de los escarpes de falla. Este índice muestra el balance entre los procesos erosivos y los procesos tectónicos que tienden a formar frentes de montaña rectos coincidentes con bordes de falla activos (Keller y Pinter, 2002). El valor se obtiene por medio de la relación entre la longitud del frente de montaña Lfm y la longitud de la línea recta que une los dos extremos del frente montañoso Ls , por lo tanto: $\mathrm{S}_{\mathrm{fm}}=\mathrm{L}_{\mathrm{fm}}$ 
Hernán Porras-Espinoza, Carolina Fallas-Salazar, Kevin Stanlyn Sarmiento, Mario Arroyo-Solorzano. Respuesta geomórfica de los ríos Tulín y Turrubaritos a la deformación asociada

la falla Angostura, antearco central de Costa Rica

$/ \mathrm{L}_{\mathrm{s}}$ (Bull, 2007). Por lo general, los valores de este índice oscilan entre 1 y 3. Mientras más cercano esté el Sfm de 1.0, más expresión morfológica tendrá, puesto que será más recto y definido, indicando que los procesos tectónicos predominan sobre los erosivos

Los valores calculados de la sinuosidad del frente de montaña $S_{f m}$ es para el escarpe 1 , de 1.08 donde $\mathrm{L}_{\text {fm }}$ (longitud del frente de la montaña a lo largo de la unión montaña-piemonte) es $5437 \mathrm{~m}$, mientras que $\mathrm{L}_{\mathrm{s}}$ (longitud de línea recta del frente) es $5015 \mathrm{~m}$ (Figura 5). Para el escarpe 2, el índice $\mathrm{S}_{\mathrm{fm}}$ tiene un valor de 1,27 , siendo el $\mathrm{L}_{\mathrm{fm}}$ de $7857 \mathrm{~m}$ y el $\mathrm{L}_{\mathrm{s}} 6172 \mathrm{~m}$. Los valores medios del índice $\mathrm{S}_{\mathrm{fm}}$ son bajos a lo largo de la falla Angostura, lo que sugiere que estos frentes de montaña son tectónicamente activos o las rocas que componen el escarpe tienen una alta resistencia a la erosión (Figura 5).

Figura 5: Frentes de montaña para los escarpes de la falla Angostura, en rojo se muestra el $\mathrm{L}_{\mathrm{fm}}$, en negro el $\mathrm{L}_{\mathrm{sm}}$. en amarillo el río Tulín señalado el tramo del río axial. Línea blanca representa el perfil longitudinal a-b.

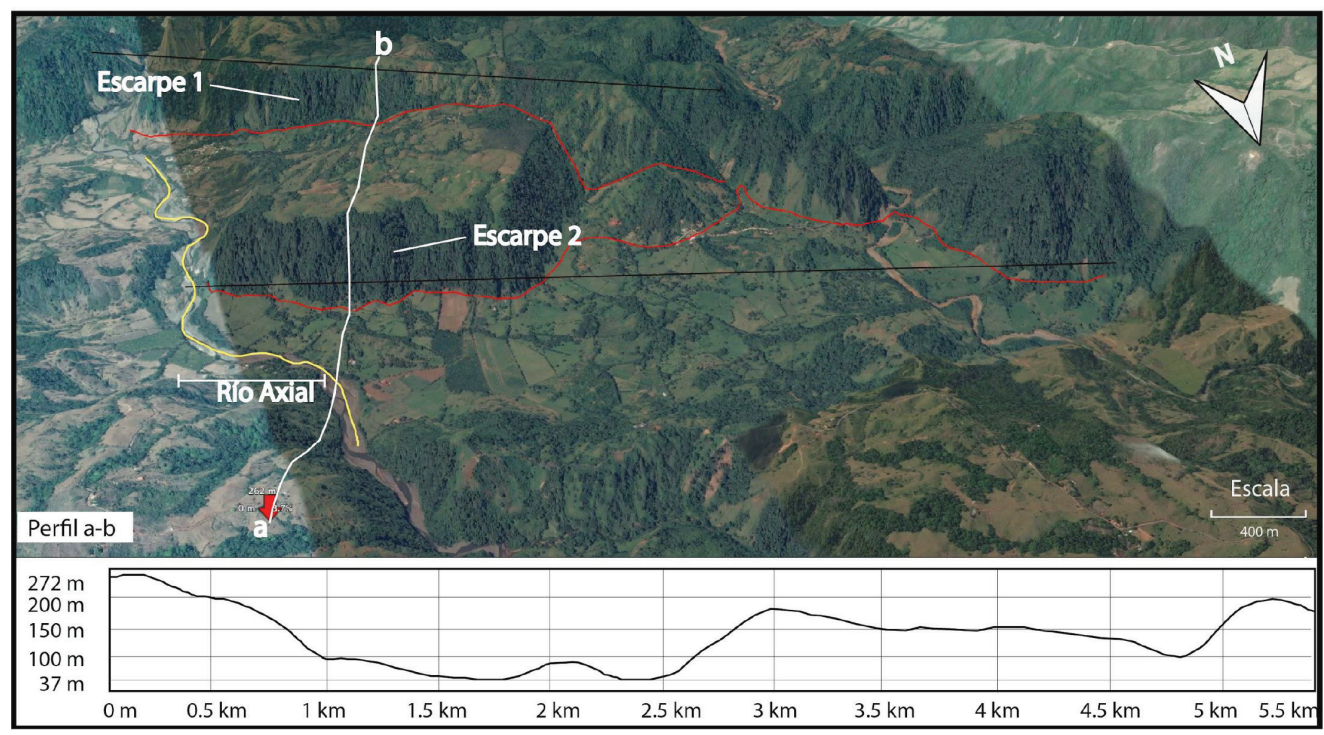

Fuente: Figura elaborada por los autores. Imagen tomada de Google Earth ${ }^{\mathrm{TM}}$.

Los valores máximos medidos a lo largo de la Falla Angostura tienen una pendiente promedio de $29.4^{\circ}$ y una altura promedio de $193 \mathrm{msnm}$, los cuales fueron estimados mediante el perfil a-b que se observa en la Figura 5. 
Hernán Porras-Espinoza, Carolina Fallas-Salazar, Kevin Stanlyn Sarmiento, Mario Arroyo-Solorzano. Geomorphic response of the rivers Tulin and Turrubaritos to the deformation associated with the angostura fault, a Central Forearc in Costa Rica

Las facetas triangulares, que consisten en rocas del basamento Cretácico en el lado oriental (alrededor de San Antonio de Tulín) (Figura 6), muestran valores de pendiente promedio máximos de $45^{\circ}$, mientras que el descenso del vector de deslizamiento superficial es de $5.1^{\circ}$ (medido en el campo). Las alturas de la faceta en proporción a los valores de la pendiente también alcanzan valores máximos a lo largo de esta zona (Figura 6).

Figura 6. Facetas triangulares a lo largo del escarpe de la Falla Angostura. Perfiles a-b y c-d en blanco representan el Lf, la topografía de las facetas y en rojo se representa el Ls, la longitud lineal de las facetas.

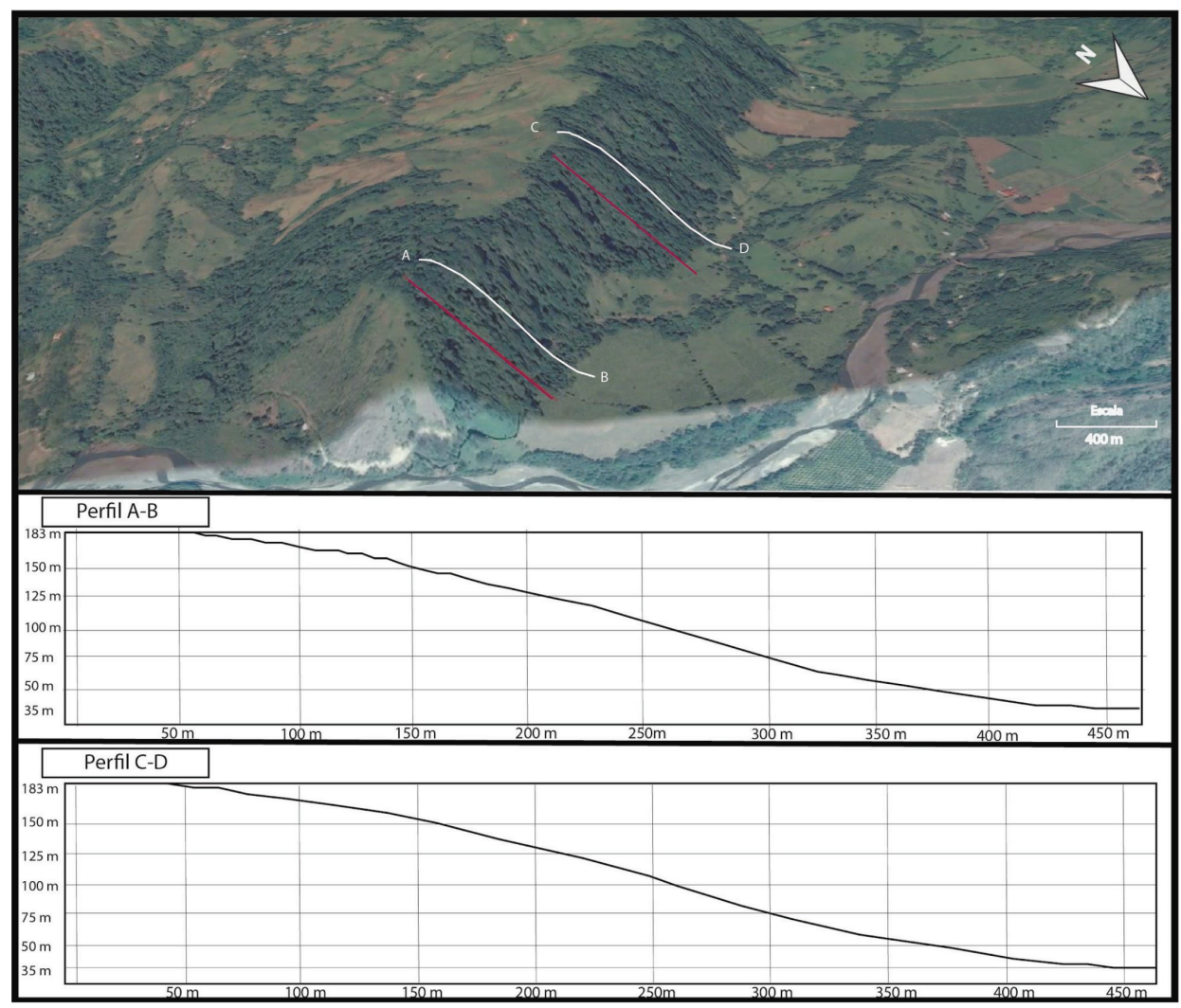

Fuente: figura elaborada por los autores. La imagen lo los perfiles topográficos son tomados de Google Earth ${ }^{\mathrm{TM}}$.

La pendiente y la altura de la faceta más alta a lo largo de la zona indican que la tasa de rechazo de la falla es claramente mayor que el 
desarrollo del suelo o la erosión de la ladera. Los valores de la pendiente y la altura disminuyen regularmente hacia los bordes donde la falla presenta una geometría escalonada (Figura 6). Como se muestra en la figura 6, la altura media de la faceta es una función lineal de la pendiente de la faceta. La proporción de facetas a lo largo del frente de montaña (Lf / Ls, donde Lf es la topografía de las facetas y Ls la longitud lineal de las facetas) se mide como 1.17 para el perfil a-b y como 1.16 para el perfil c-d a lo largo de la falla Angostura. Los datos obtenidos están representados por valores altos en las terminaciones occidental y oriental de la falla Angostura (Figura 6). Las facetas medidas varían en longitud entre 150 y 160 m (155 m de media) (Figura 6); la distribución de la longitud de la faceta es consistente con la distribución de la altura de la faceta a lo largo del frente de la montaña.

En general, el espaciamiento real de las cuencas depende de su redondez y alargamiento; cuanto más circular es una cuenca, menos eficientemente llena el espacio y las facetas triangulares serán, por lo tanto, más amplias (Burbank y Anderson, 2001).

\section{Análisis del patrón de drenaje}

\section{Río axial y sistema de drenaje de los ríos Tulin y Turrubaritos}

Los drenajes de los ríos Tulin y Turrubaritos se ven fuertemente influenciados por las condiciones fallamiento en el antearco externo de Costa Rica. Esta condición aunada a los procesos erosivos interviene dentro de la dinámica de dichos ríos.

Los ríos Tulin y Turrubaritos, se pueden subdividir en múltiples segmentos, según la orientación de la respuesta hidrológica de los mismos. En la Figura 7, se evidencia la subdivisión para cada río y como esta se relaciona con zonas de levantamiento y otras de subsidencia. Una zona de levantamiento relativo produce un cambio que marca la diferencia entre los segmentos 5 y 6 de ambos ríos, donde se modifica la orientación de los mismos y se da la incisión del cauce entre dicho levantamiento o zona de "uplift". 
Hernán Porras-Espinoza, Carolina Fallas-Salazar, Kevin Stanlyn Sarmiento, Mario Arroyo-Solorzano. Geomorphic response of the rivers Tulin and Turrubaritos to the deformation associated with the angostura fault, a Central Forearc in Costa Rica

Figura 7: Segmentación de la respuesta hidrológica de los ríos Tulin y Turrubaritos a los procesos de levantamiento y subsidencia de la cuenca.

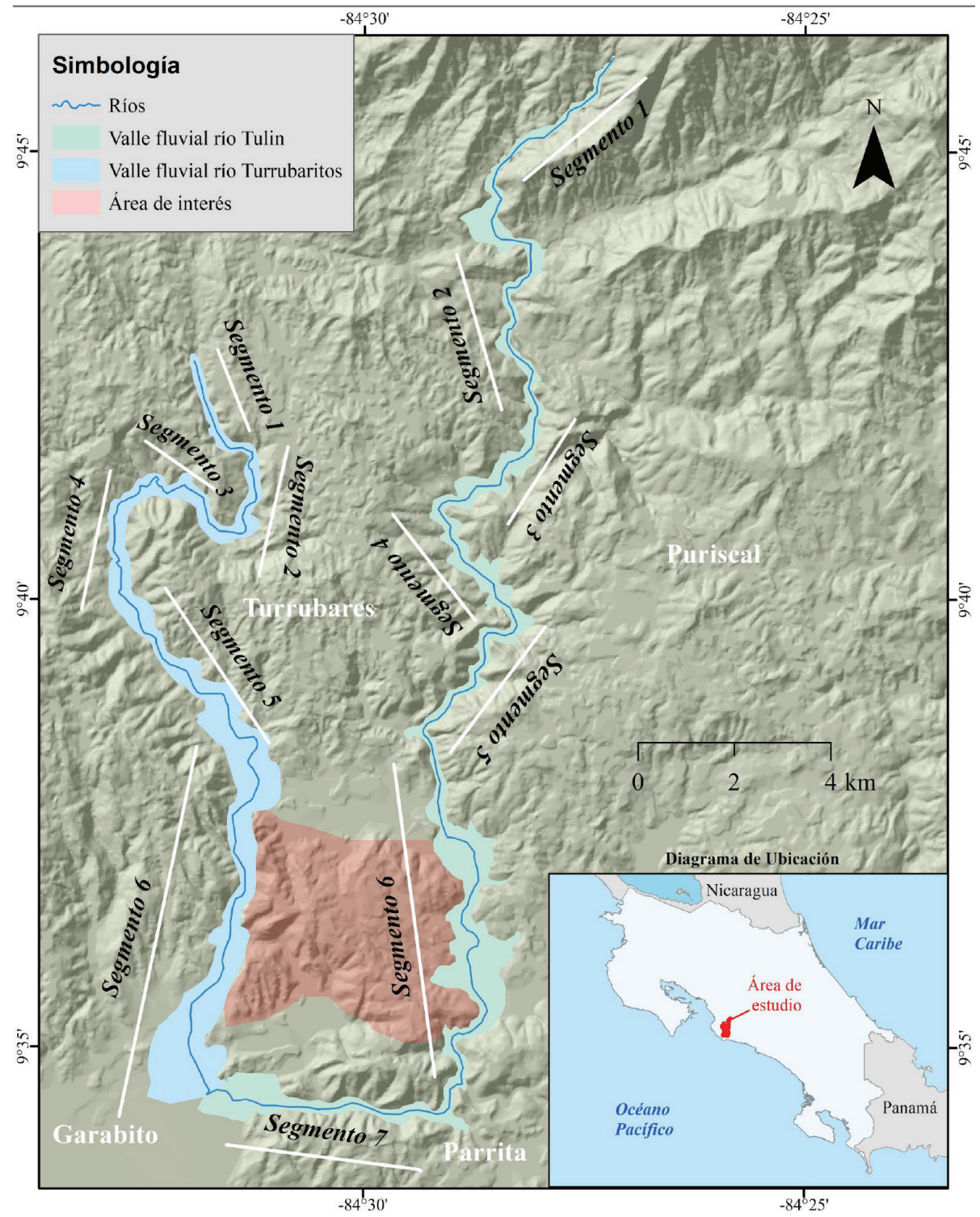

Según Holbrook y Schumm (1999), la deformación que afecta la superficie del suelo también deforma la topografía debajo del curso del río y este efecto puede causar la convexidad de las terrazas y del fondo del 
Hernán Porras-Espinoza, Carolina Fallas-Salazar, Kevin Stanlyn Sarmiento, Mario Arroyo-Solorzano. Respuesta geomórfica de los ríos Tulín y Turrubaritos a la deformación asociada

la falla Angostura, antearco central de Costa Rica

valle. El principal efecto de esta deformación se observa en el perfil del canal en relación con el gradiente de valle promedio regional. La Figura 8 , muestra los perfiles longitudinales tanto del cauce como de las terrazas de los segmentos 5, 6 y 7 del río Tulin y 6 y 7 del río Turrubaritos. Los perfiles longitudinales, exhiben una inclinación promedio de 5,3\% para el río Tulin y de 3,8 \% para el Turrubaritos.

Figura 8: Perfiles longitudinales de los cauces y terrazas de los ríos Tulín (A) y Turrubaritos (B). Figura elaborada por los autores.

A) Río Tulín

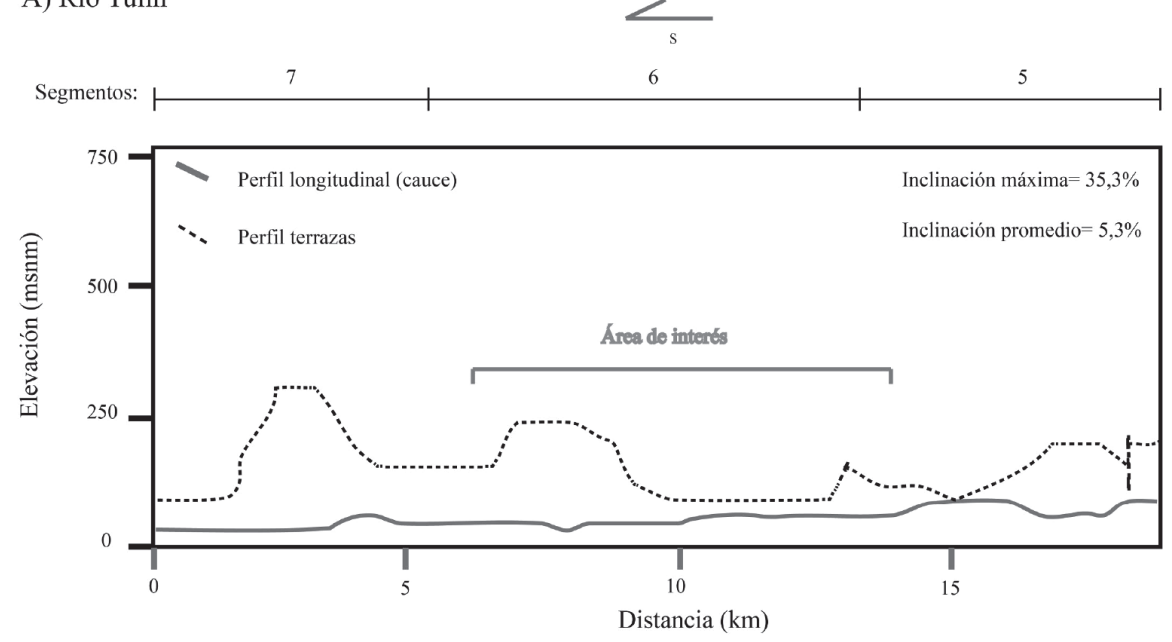

B) Río Turrubaritos

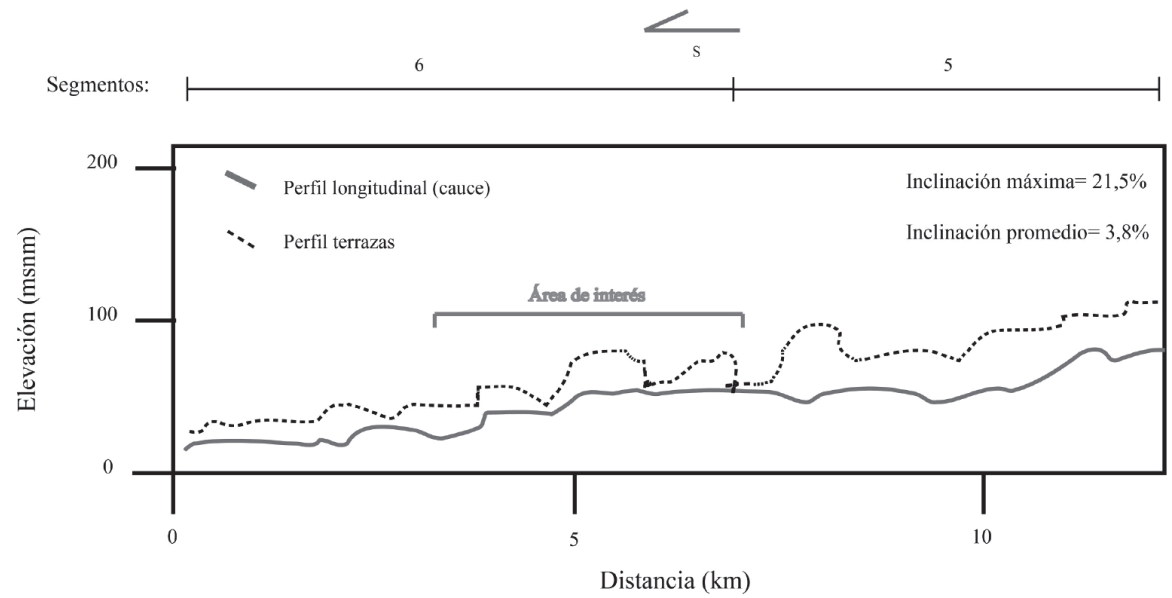


Hernán Porras-Espinoza, Carolina Fallas-Salazar, Kevin Stanlyn Sarmiento, Mario Arroyo-Solorzano. Geomorphic response of the rivers Tulin and Turrubaritos to the deformation associated with the angostura fault, a Central Forearc in Costa Rica

Los perfiles longitudinales de los cauces muestran una geometría convexa no representativa de un perfil de equilibrio, la misma que se aprecia en buena parte de ambos ríos (Figura 8). En el caso del río Tulín (Figura 8.A), esto se observa justo antes de entrar en el segmento 6 , donde se ve un patrón convexo del cauce, que puede evidenciar el efecto de la Falla Angostura sobre el cauce. En el caso del río Turrubaritos (Figura 8.B), se muestra cierta convexidad en el segmento 6 .

En la cuenca Tulin, un río axial está representado por la corriente del río Tulin que sigue la tendencia de la falla Angostura (Figura 6). Los gradientes del canal (pendiente) y la sinuosidad del río axial se calcularon por cada $10 \mathrm{~m}$ de elevación en la pared colgante de la falla. A partir de los cálculos morfométricos, se observó que los canales tienen una gran sinuosidad y pronunciados gradientes de canal.

De acuerdo con los cálculos, el gradiente de canal medio del río axial mantiene una pendiente generalmente uniforme de 7.9 (\%) con una sinuosidad del canal de 1.06, determinada como la relación de la longitud total del canal entre la longitud en línea recta entre cada tramo del mismo, a lo largo de la cuenca de Tulin, donde se forma el río axial. Estos valores indican que el río axial tiene un patrón de transición serpenteante a trenzado, que representa el patrón de canal de carga mixta según la clasificación de tipo de canal (Schumm, 1986).

En la parte oriental de la Cuenca de Tulin, el drenaje axial del rio Tulin fluye hacia el oeste con un gradiente de canal de $6.6(\%)$ y una sinuosidad del canal de 1.151 (Figura 5). Los valores de gradiente de canal son más constantes en el centro de la cuenca del rio Tulin, antes de la confluencia con la corriente de Turrubaritos, con un gradiente de canal no mayor al 3 (\%) y una sinuosidad de canal siempre cercana al 1.

Además, se han observado algunos paleocanales y paleolagunas en el lado suroeste del río axial (Figura 9), entre el cauce actual del río y el escarpe de falla, lo que indica la migración lenta del río Tulin (e.g. Alexander y Leeder, 1987) en la pared colgante.

Para identificar el proceso de migración del río, se midieron las posiciones relativas de las lagunas y el canal principal (Figura 9). La Figura 9 , muestra los efectos de un evento de inclinación perpendicular a un canal serpenteante. Cuando se produce un evento de inclinación repentino, el canal se desplaza con una avulsión repentina, dejando cicatrices de meandro 
Hernán Porras-Espinoza, Carolina Fallas-Salazar, Kevin Stanlyn Sarmiento, Mario Arroyo-Solorzano. Respuesta geomórfica de los ríos Tulín y Turrubaritos a la deformación asociada

la falla Angostura, antearco central de Costa Rica

orientadas al azar hacia la dirección de hundimiento relativo. Las ubicaciones de los paleocanales y el canal moderno del río axial implican la migración de corrientes hacia el noreste, $\mathrm{y}$ tales datos indican poca inclinación hacia atrás del piso de la cuenca. En términos generales el rio axial parece estar alejándose del escarpe de falla.

Figura 9: a) Imagen satelital del área de estudio del año 2012 tomada de Google Earth ${ }^{\mathrm{TM}}$. b) se muestra paleocanales y paleolagunas del rio tulin.

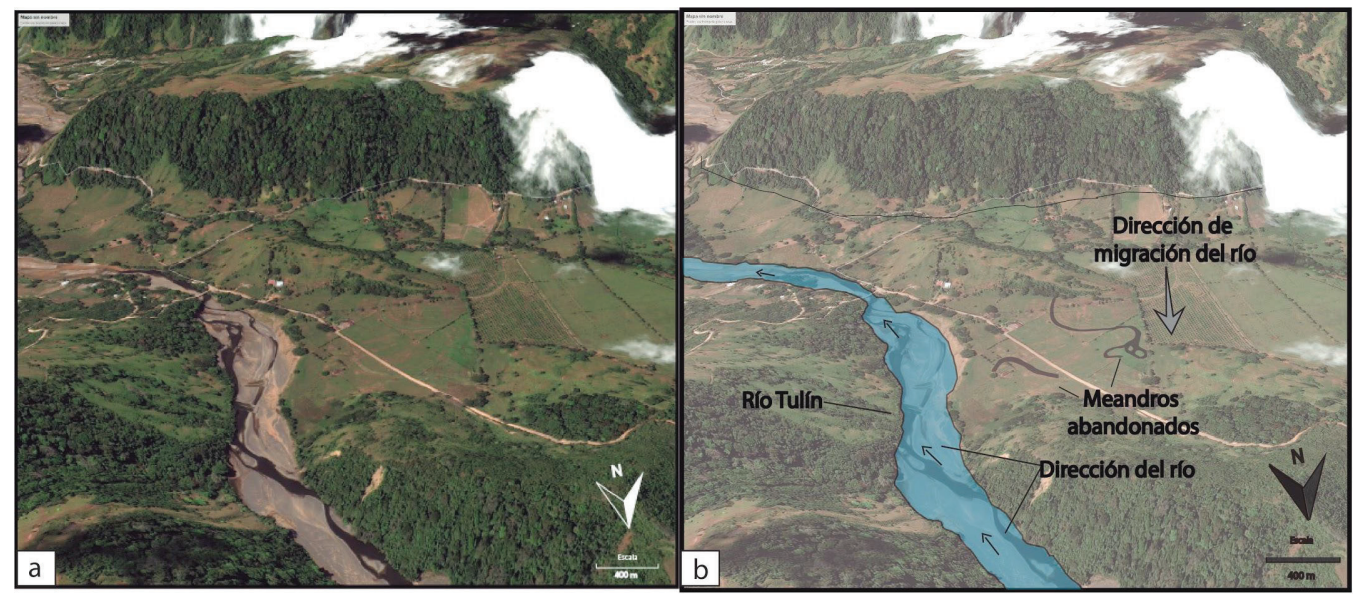

Fuente: imagen satelital tomada de Google Earth ${ }^{\mathrm{TM}}$.

\section{Discusión}

Cerca del centro de las trazas de la falla, los lineamientos indican un deslizamiento de aproximadamente noroeste-sureste, que es compatible con la extensión noreste-suroeste regional en el antearco externo interpretado por Barbosa et al. (1995) a partir de las líneas sísmicas. La geometría de la falla normal, sugiere un sistema de relevo mediante una rampa, la cual estaría ubicada en el poblado de San Antonio de Tulín (Figura 10). 
Hernán Porras-Espinoza, Carolina Fallas-Salazar, Kevin Stanlyn Sarmiento, Mario Arroyo-Solorzano. Geomorphic response of the rivers Tulin and Turrubaritos to the deformation associated with the angostura fault, a Central Forearc in Costa Rica

Figura 10: a) Escarpes de la falla Angostura. b) Esquema del sistema de relevo mediante una rampa de la Falla Angostura.
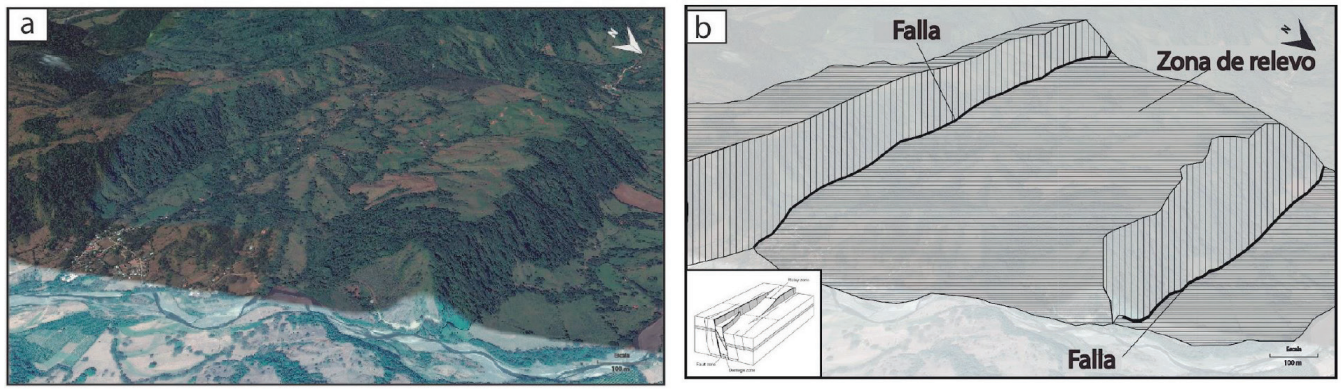

Fuente: imagen satelital tomada de Google Earth ${ }^{\mathrm{TM}}$.

De acuerdo con las observaciones de campo y el mapeo geológico en el área, se identifican los enlaces entre estos segmentos de fallas y las áreas de sobrepaso, la cual muestra una geometría de rampa de relevo típica (Figura 10). Las rampas de relevo, las fallas normales y las zonas de falla de transferencia también son típicas en un entorno extensional. $\mathrm{Mu}$ chos segmentos de fallas normales individuales paralelas / sub-paralelas tienen una tendencia a unirse entre sí, y este enlace entre fallas en-échelon permite la consiguiente formación de una estructura de rampa de relevo. Las fallas (sub) paralelas permiten el desarrollo y la ruptura de rampas de relevo entre los segmentos de la falla Angostura. Muchos sistemas de fallas normales, caracterizados por segmentos sobrepasadas y vinculados, muestran escarpes continuos con rastros de zigzag.

Con los resultados obtenidos, se plantea que las estructuras asociadas a la esta falla, fueron las responsables en muy buena medida de la deformación y de las morfologías escarpadas, así como de los parámetros obtenidos a partir del análisis del drenaje de los ríos Tulín y Turrubaritos.

La interpretación realizada en este estudio con referencia a los paleocanales y las paleolagunas muestra una migración lateral del río axial a la región distal del escarpe de la falla Angostura. Por lo tanto, la migración del canal lateral pudo haber progresado hacia el escarpe en el pasado, como respuesta a la inclinación de la llanura de la cuenca en dirección suroeste, y, probablemente, fue el resultado de la actividad de la falla Angostura. Esta migración a un sector distal del escarpe de falla es un indicador de quietud tectónica. 
Hernán Porras-Espinoza, Carolina Fallas-Salazar, Kevin Stanlyn Sarmiento, Mario Arroyo-Solorzano. Respuesta geomórfica de los ríos Tulín y Turrubaritos a la deformación asociada

la falla Angostura, antearco central de Costa Rica

Los perfiles longitudinales sugieren que la tasa de incisión del canal es mayor que la tasa de elevación, o que las corrientes son demasiado viejas para haber incidido lo suficiente. El análisis de los perfiles longitudinales de los canales de la corriente principal denota que el punto de corte principal a lo largo del frente de montaña es el resultado de la poca o nula actividad cuaternaria de la falla Angostura. Esta posiblemente se desarrolló durante un periodo extensional en el Mioceno inferior, justo cuando la Formación Roca Carballo (rocas sedimentarias del Neógeno, Figura 2) era depositada.

Este tipo de deformación no está asociada, por lo tanto, al periodo contraccional ocurrido durante el Mioceno medio y parece no estar influenciado por el periodo transpresivo actual. La asimetría de las cuencas principales de corrientes no indican que el valle se incline hacia el suroeste. Este resultado indica que la falla Angostura no ha tenido actividad reciente y por lo tanto la actividad de esta no ha tenido un impacto significativo en la evolución del paisaje actual.

Las geoformas asociadas a la actividad de la falla, tales como los escarpes y la rampa de relevo pueden ser estructuras fosilizadas en el antearco central. En este contexto, la litología del basamento funge como agente prominente para el desarrollo de cuencas de drenaje. La resistencia de las rocas del basamento que están predominantemente compuestas de basaltos impide el desarrollo posterior de muchas facetas triangulares bien desarrolladas a lo largo del frente montañoso de San Antonio de Tulin. De manera similar, las cuencas de drenaje en la zona están compuestas por varias unidades litológicas relativamente débiles, tales como areniscas y lutitas de la Formación Punta Carballo (rocas sedimentarias del Neógeno, Figura 2). Las cuencas de drenaje que se han desarrollado en las rocas basálticas resistentes tienen un relieve de cuenca más empinado que las formadas en rocas sedimentarias menos resistentes.

Por lo tanto, no se encontró evidencia mensurable que permita afirmar que la falla se encuentra activa. En su lugar, aspectos como la migración del cauce del río lejos de los escarpes asociados a la falla, las facetas triangulares y la poca o nula actividad sísmica en el sector en específico de esta falla, dan pie a pensar en que la falla actualmente no presenta actividad. 


\section{Conclusiones}

La cuenca media-baja de los ríos Tulin y Turrubaritos, del antearco externo de Costa Rica, corresponde a una región con desplazamientos, levantamientos y/o hundimientos diferenciales que afectan los sistemas fluviales activos de dichos ríos. Las evidencias más claras de esto se encuentran en el análisis geomórfico y los patrones de drenaje que moldean los respectivos cauces.

El trabajo de campo y el análisis geológico-geomorfológico de la Falla Angostura en estos cauces, indica que los enlaces entre estos segmentos de fallas y las áreas de sobrepaso muestran una geometría de rampa de relevo típica. Además, se asocia un régimen de esfuerzos de tipo extensional, con lo cual se determina que la falla presenta un componente principalmente de tipo Normal, con una geometría lístrica y un deslizamiento casi puro, un rumbo que sigue predominantemente una dirección NorOeste-SurEste y un plano de inclinación medio de aproximadamente $45^{\circ}$ hacia el noreste.

La zona escarpada detallada en esta investigación, se presenta como un sector de levantamiento en los patrones de drenaje de ambos cauces estudiado. Los perfiles longitudinales de dichos cauces, muestran una geometría convexa no representativa de un perfil de equilibrio. Los puntos anteriores pueden evidenciar el efecto de la falla Angostura sobre el cauce, sin embargo, esto se asocia más a una paleotectonismo que se desarrolló probablemente en el Mioceno Inferior-Medio.

El índice de sinuosidad del frente de montaña $\left(\mathrm{S}_{\mathrm{fm}}\right)$ para el escarpe 1 es de 1.08 y para el escarpe 2, de 1.27, siendo estos valores bajos a lo largo de la falla Angostura y asociadas en este caso, a que las rocas que componen el escarpe tienen una alta resistencia a la erosión. Las facetas triangulares estudiadas, presentan valores de 1,17 y 1,16, mostrándose muy desarrolladas, esto sumado a la poca actividad sísmica en el sector, reflejan la no actividad reciente de la falla. La situación anterior es reafirmada por los paleocanales y las paleolagunas, que muestra una migración lateral del río axial a la región distal del escarpe de la Falla Angostura, lo cual también es un indicador de quietud tectónica en la actualidad.

Es importante reafirmar, que la conclusión esencial refiere que las geoformas asociadas a la actividad de la falla Angostura, tales como los escarpes y la rampa de relevo pueden ser estructuras fosilizadas en el 
Hernán Porras-Espinoza, Carolina Fallas-Salazar, Kevin Stanlyn Sarmiento, Mario Arroyo-Solorzano.

Respuesta geomórfica de los ríos Tulín y Turrubaritos a la deformación asociada

la falla Angostura, antearco central de Costa Rica

antearco central de Costa Rica. En este contexto, la litología del basamento funge como el principal agente prominente para el desarrollo de cuencas de drenaje y sus respectivos patrones que se presentan hoy día y que se han desarrollado desde el Cuaternario.

\section{Referencias}

Arroyo, M., Godínez, K., y Linkimer, L. (2017). Completitud del catálogo de la Red Sismológica Nacional de Costa Rica durante 1975-2014. En: Boletín de Geología. 39(3): 87-98.

Astorga, A., Fernandez, J., Barboza, G., Campos, L., Obando, J., Aguilar, A. y Obando, L. (1991). Cuencas sedimentarias de Costa Rica: Evolución geodinámica y potencial de hidrocarburos. En: Rev. Geol. Amér. Central, 1(13), 25-59.

Alexander, J. y Leeder, M. R. (1987). Active tectonic control on alluvial architecture. In: Recent Developments in Fluvial Sedimentology (Ed. by F.G. Ethridge, R. M. Flores and M. D. Harvey). En: Society of Economic Paleontologists and Mineralogists Special Publication, 39, 243-252.

Arias, O. (2013). Redefinición de la formación Tulín (MaastrichtianoEoceno inferior) del Pacífico Central de Costa Rica. En: Rev. Geol. Amér. Central. 1 (28), 47-68.

Arias, O. y Denyer, P. (1991). Estructura geológica de la región comprendida en las Hojas Topográficas Abra, Caraigres, Candelaria y Río Grande de Costa Rica. En: Rev. Geol. Amér. Central. 1. (12), 67 - 71. Barboza, M., Carpenter, S. y Roche, L. (1995). Prediction of traffic noise: A screening technique. J. of Air and Waste Manage. En: Assoc. 45, 703- 708.

Burbank, D. y Anderson, R. (2001). Tectonic Geomorphology. Main Street, Malden, USA: Blackwell Science.

Bull, W. B. (2007). Tectonic geomorphology of mountains: A new approach to paleoseismology. Oxford:Blackwell. doi: 10.1002/9780470692318 Bundschuh, J. y Alvarado, G. (2007). Central America: Geology, Resources and Hazamis. Vol. 2. London: Taylor and Francis.

Denyer, P., Montero, W., \& Alvarado, G. (2003). Atlas Tectónico de Costa Rica. San José, Costa Rica: Universidad de Costa Rica.

Bird, P. (2003). An updated digital model of plate boundaries. En: Geochem. Geophys. Geosyst. 4 (3), 1027, doi:10.1029/2001GC000252, 2003. 
Hernán Porras-Espinoza, Carolina Fallas-Salazar, Kevin Stanlyn Sarmiento, Mario Arroyo-Solorzano. Geomorphic response of the rivers Tulin and Turrubaritos to the deformation associated with the angostura fault, a Central Forearc in Costa Rica

DeMets, C. (2001). A new estimate for present-day Cocos-Caribbean plate motion: implications for slip along the Central American volcanic arc. En: Geophys. Res. Lett. 28, 4043-4046.

DeMets, C.,Gordon, RyArgusD.(2010).Geologicallycurrentplatemotions.En: Geophys. J. Int., 181(1), 1-80, doi:10.1111/j.1365-246X.2009.04491.x. Denyer, P. y Arias, O. (1991). Estratigrafía de la Región Central de Costa Rica. En: Rev. Geol. Amér. Central. 12, 1-59.

Denyer, P y Alvarado, G. (2007). Mapa Geológico de Costa Rica, 2007. Escala 1:450 000. Librería Francesa S.A.

Fisher, D., Gardner, T. Sak, P., Sánchez, J., Murphy, K. y Vannucchi, P. (2004). Active thrusting in the inner forearc of an erosive convergent margin, Pacific coast, Costa Rica. En: Tectonics, 23, TC2007, doi:10.1029/2002TC001464.

Gardner, T. W., Marshall, J., Merrits, D., Bee, B., Burgette, R., Burton, D., Cooke, J., Kehrwals, N., Protti, M., Fisher, D. y Sak P. (2001). Holocene forearc block rotation in response to seamount subduction, southeastern Peninsula de Nicoya, Costa Rica. En: Geology. 2, (29), $151-154$.

Godínez, K., Arroyo, M. y Linkimer, L. (2018). Distribución geográfica de los sismos contenidos en el catálogo de la Red Sismológica Nacional de Costa Rica. En: Rev. Geográfica de América Central, "pendiente de publicación".

Keller, E. (1986). Investigation of active tectonics: Use of surficial Earth processes. In R. E. Wallace (Ed.), Washington, DC: National Academy Press. Studies in Geophysics. Active tectonics. (pp. 136-147).

Keller, E. A., y Pinter, N. (2002). Active tectonics. Earthquakes, uplift, and landscape (362 pp.). Upper Saddle River, NJ: Prentice Hall.

LaFemina, P., Dixon, H., Govers,T., Norabuena, E., Turner, H., Saballos, A., Mattioli, G., Protti, M. y Strauch W. (2009). Fore-arc motion and Cocos Ridge collision in Central America. En: Geochem. Geophys. Geosyst., 10, 1525-2027, doi:10.1029/2008GC002181.

Morell, K., Kirby, E., Fisher, D. y Van Soest, M. (2012): Geomorphic and exhumational response of the Central American Volcanic Arc to Cocos Ridge subduction. En: Journ. Geophys, Res. 117, doi:10.1029/2011JB008969. 
Hernán Porras-Espinoza, Carolina Fallas-Salazar, Kevin Stanlyn Sarmiento, Mario Arroyo-Solorzano. Respuesta geomórfica de los ríos Tulín y Turrubaritos a la deformación asociada

la falla Angostura, antearco central de Costa Rica

Mescua, J., Durán, P., Porras, H., Giambiagi, L., Moor, M., Cascante, M., Salazar, E., Protti, M. y Poblete, F. (2017). Middle to Late Miocene Contractional Deformation in Costa Rica Triggered by Plate Geodynamics. En: Tectonics. 36, doi: 10.1002/2017TC004626.

Mann, P. (2007), Geologic and tectonic development of the Caribbean plate boundary in northern Central America. En:Geological Society of America, Boulder, CO, Special Paper, 179 pp.

Protti, M., Güendel, F. y K. McNally (1995), Correlation between the age of the subducting Cocos Plate and the geometry of the Wadati-Benioff zone under Nicaragua and Costa Rica, in Geologic and Tectonic Development of the Caribbean Plate Boundary in Southern Central America, edited by P. Mann, pp. 309-326. En: Geol. Soc. Am. Spec. Pap. 295, Boulder, Colo.

Norabuena, E., Leffler, L., Mao, A., Dixon, T., Stein, S., Sacks, S. y Ellis., M. (1998): Space geodetic observation of Nazca-South America convergence along the central Andes. En: Science, 279, 358-362.

Sak, P., Fisher, D., y Gardner T. (2004). Effects of subducting seafloor roughness on upper plate vertical tectonism: Osa Peninsula, Costa Rica. En: Tectonics, 23, TC1017, doi:10.1029/2002TC001474.

Sak, P. B., Fisher, D., Gardner, T., Marshall, J., y LaFemina P. (2009). Rough crust subduction, forearc kinematics, and Quaternary uplift rates, Costa Rican segment of the Middle America Trench. En: Geol. Soc. Am. Bull., 121(7/8), 992-1012, doi:10.1130/B26237.1.

Seeber, L. y Gornitz, V. (1983). River profiles along the Himalayan arc as indicators of active tectonics. En: Tectonophysics,. 92,. 335-367.

Sitchler, J. C., Fisher, D., Gardner, T y Protti, M. (2007). Constraints on inner forearc deformation from balanced cross sections, Fila Costeña thrust belt, Costa Rica. En: Tectonics, 26, TC6012, doi:10.1029/2006TC001949.

Schumm, A. S. (1986). Alluvial river response to active tectonics. En: R. E. Wallace (Ed.), Active tectonics (pp.80-94). Washington, DC: National Academy Press. Studies in Geophysics.

Saginor, I., Gazel, E., Condie, C. y Carr, M.J. (2013). Evolution of geochemical variations along the Central American volcanic front. En: Geochem. Geophys. Geosyst. 14(10), 4504-4522, doi:10.1002/ ggge.20259. 
